\title{
Prevalência e correlação das comorbidades por idade e sexo dos óbitos por COVID-19 no estado de Sergipe - Brasil: Parte I
}

\author{
Prevalence and correlation of comorbidities by age and sex of COVID-19 deaths in \\ Sergipe - Brazil state: Part I
Prevalência y correlación de las comorbilidades por edad y sexo de lãs muretas de COVID-19 em estado de Sergipe - Brasil: Parte I

Kathlen Cruz Almeida1', Carlos Henrique da Silva Marcelino1, Laiany Lima da Cruz¹, Luís Augusto Silva Rocha ${ }^{1}$, Fabiana Conceição de Oliveira Santos Falcão ${ }^{1}$, Júlia Caroline Santos ${ }^{1}$, Sônia Oliveira Lima $^{1,2}$, Edna Aragão Farias Cândido ${ }^{1,2 *}$.

\section{RESUMO}

Objetivo: Evidenciar a prevalência e sua correlação com as comorbidades por idade e sexo dos óbitos confirmados por COVID-19 no estado de Sergipe-Brasil. Métodos: O estudo retrospectivo e transversal foi realizado em 28/06/2020 com dados da Vigilância Epidemiológica de Sergipe, analisados pelo teste Qui-quadrado com nível de significância de $p>0.05$. Foram 620 óbitos com 73,06\% associados a comorbidades. Resultados: O sexo masculino (53,02\%) prevaleceu Hipertensão (30,59\%), seguido de Diabetes (28,02\%) e Cardiopatias (10,8\%). No sexo feminino $(46,08 \%)$ a prevalececia foi semelhante, Hipertensão (30,95\%), Diabetes (28,37\%) e Cardiopatias (12,32\%). Os óbitos acima de 50 anos influenciaram moderadamente $(r=0,65 ; p=0,001)$ e fortemente $(r=0,8 ; p=0,001)$ acima dos 60 para os óbitos, independentemente das comorbidades. E tanto no sexo masculino $(r=0,18 ; p=0,003)$ como feminino $(r=0,23 ; p=0,001)$ essa correlação foi fraca para Hipertensão. Assim como para a associação de Hipertensão e Diabetes no sexo masculino $(r=0,13 ; p=0,043)$ e feminino $(r=0,16 ; p=0,018)$. Conclusão: Em Sergipe tanto os homens como as mulheres morrem de COVID-19, principalmente acima dos 60 anos e com comorbidades associadas como a hipertensão arterial.

Palavras-chave: Pandemia, COVID-19, Comorbidade, Óbito.

\section{ABSTRACT}

Objective: To evidence the prevalence and its correlation with comorbidities by age and sex of the deaths confirmed by OVID-19 in the state of Sergipe-Brazil. Methods: The retrospective and cross-sectional study was conducted on 06/28/2020 with data from Sergipe Epidemiological Surveillance, analyzed by the Chi-square test with a significance level of $p>0.05$. There were 620 deaths with $73.06 \%$ associated with comorbidities. Results: The male gender $(53.02 \%)$ prevailed hypertension (30.59\%), followed by diabetes (28.02\%) and heart disease (10.8\%). In females (46.08\%) the prevalence was similar, Hypertension (30.95\%), Diabetes (28.37\%) and Heart Diseases (12.32\%). The deaths above 50 years influenced moderately $(r=0.65 ; p=0.001)$ and strongly $(r=0.8 ; p=0.001)$ above 60 for deaths, regardless of comorbidities. And in both males $(r=0.18 ; p=0.003)$ and females $(r=0.23 ; p=0.001)$ this correlation was weak for hypertension. As for the association of Hypertension and Diabetes in males $(r=0.13 ; p=0.043)$ and females $(r=0.16 ; p$ $=0.018$ ). Conclusion: In Sergipe both men and women die of COVID-19, especially over 60 years with associated comorbidities such as arterial hypertension.

Keywords: Pandemic, COVID-19, Comorbidity, Death.

\section{RESUMEN}

Objetivo: Poner en evidencia la prevalencia y su correlación con las comorbilidades por edad y sexo de las muertes confirmadas por OVID-19 en el estado de Sergipe-Brasil. Métodos: El estudio retrospectivo y transversal se realizó el 28/06/2020 con datos de la Vigilancia Epidemiológica de Sergipe, analizados por la prueba de Chi cuadrado con un nivel de significación de p>0.05. Hubo 620 muertes con un 73,06\% asociado a comorbilidades. Resultados: En el género masculino $(53,02 \%)$ prevaleció la hipertensión $(30,59 \%)$, seguida de la diabetes $(28,02 \%)$ y las enfermedades cardíacas $(10,8 \%)$. En las mujeres $(46,08 \%)$ la prevalencia fue similar, la hipertensión $(30,95 \%)$, la diabetes $(28,37 \%)$ y las enfermedades cardíacas (12,32\%). Las muertes por encima de 50 años influyeron moderadamente $(r=0,65 ; p=0,001)$

\footnotetext{
1 Universidade Tiradentes (UNIT), Aracaju - SE. *E-mail: edna_candido@email.itp.org.br
}

2 Instituto de Tecnologia e Pesquisa da Universidade Tiradentes (UNIT), Aracaju - SE. 
y fuertemente $(r=0,8 ; p=0,001)$ por encima de 60 para las muertes, independientemente de las comorbilidades. $Y$ tanto en los hombres $(r=0,18 ; p=0,003)$ como en las mujeres $(r=0,23 ; p=0,001)$ esta correlación fue débil para la hipertensión. En cuanto a la asociación de la Hipertensión y la Diabetes en los hombres $(r=0,13 ; p=0,043)$ y las mujeres $(r=0,16 ; p=0,018)$. Conclusión: En Sergipe tanto hombres como mujeres mueren de COVID-19, especialmente más de 60 años con comorbilidades asociadas como hipertensión arterial.

Palabras clave: Pandemia, VCOCID-19, Comorbilidad, Muerte.

\section{INTRODUÇÃO}

A pandemia causada pelo novo Corona vírus 2019 (COVID-19) surgiu em Wuhan na China após um surto significativo de pacientes apresentando um tipo de pneumonia de origem desconhecida. Afetando assim, grande parte dos habitantes que residiam e trabalhavam próximo ao mercado onde eram vendidos produtos alimentícios para o consumo de forma imediata (LI LQ, et al., 2020). Logo após o novo vírus descoberto, a doença foi denominada COVID-19 com base nas suas manifestações clínicas, testes sanguíneos e radiografias de tórax (JIN Y, et al., 2020).

Nomeado SARS-CoV-2 o vírus faz parte da família do Coronaviridae, o seu hospedeiro pode utilizar do receptor da Enzima Conversora de Angiotensina II (ECA2) para adentrar-se nas células, semelhante a Síndrome Respiratória Aguda Grave do coronavírus (SARS-CoV) (LIU Z, et al., 2020). Apesar de alguns animais apresentarem condições como hospedeiros do SARS-CoV-2, o processo de transmissão acontece de humano para humano (sintomáticos ou não) por meio de gotículas ou transmissão por contato, que podem permanecer suspensas até 3 horas no ar (BULUT C, et al., 2020).

A reposta pós-contaminação inclui infecção do epitélio das vias aéreas e os pneumócitos alveolares do tipo 2 (HARAPAN H, et al., 2020). Quando manifestados, os sintomas aparecem por volta do quinto ou sexto dia após período de incubação e podem durar por até 14 dias. As manifestações mais comuns são febre, tosse seca, dispneia, fadiga e a perda do olfato ou paladar, já as menos frequentes são a presença de dor e desconforto, diarreia, conjuntivite, cefaléia e erupções cutâneas na pele. Nos casos mais graves pode-se ocorrer a apresentação de dificuldade ou falta de ar, angina ou dor torácica, redução no movimento dos membros ou alterações na fala (RODRIGUES-MORALES AR, et al., 2020).

A população com comorbidades associadas aos sistemas pulmonar e cardiovascular, como a doença pulmonar obstrutiva crônica (DPOC) e doença renal aguda, apresentam-se mais propensas a infecção (GE $\mathrm{H}$, et al., 2020). As mortes, por muitas vezes, estão vinculadas a comorbidades como a Hipertensão Arterial Sistêmica (HAS) e a Diabetes Mellitus (DM), além das doenças cardiovasculares (YANG X, et al., 2020). Em Wuhan na China, das 26 mortes, 42,3\% foram em pessoas com diabetes, a prevalência da HAS foi maior quando comparada a DM (ZHANG JJ, et al., 2020; GUAN WJ, et al., 2019).

Os pacientes que são infectados apresentam alteração no número de leucócitos, bilirrubina total, creatina quinase, ferritina sérica e nos níveis de interleucina 6 . A elevação destes níveis está presente nos pacientes que não sobrevivem em comparação com os sobreviventes, além disso, aqueles indivíduos em estado mais grave apresentam redução significativa de células de defesa, como os linfócitos e as plaquetas (HENRY BM, et al., 2020).

A gravidade dos indivíduos infectados pela COVID-19 tem causado milhares de mortes, em decorrência de rápida contaminação, além de que, a disseminação do vírus pode ser observada em todo o mundo (GUPTA $R$, et al., 2020). No Brasil, a doença tem se alastrado e é causa de milhares de óbitos, a propensão de infecção àqueles com comorbidades como a HAS e DM parece ser um problema para a população do país. Segundo o Ministério da Saude do Brasil (2011), a HAS e DM fazem parte das doenças crônicas não transmissíveis de maior magnitude entre os anos de 2011 e 2022.

Com isso, essas doenças são conhecidas como principal causa de morbidade e mortalidade no Brasil (ALMEIDA ATC, et al., 2019). De acordo com Zhou F, et al. (2020), dentre os indivíduos internados, por COVID-19, em um hospital em Wuhan na China, a HAS é a comorbidade mais incidente, seguida da DM. A associação a essas comorbidades pode ser devido a condição hiper inflamatória, que desencadeia maior 
suscetibilidade ao COVID-19. Tadic M, et al., (2020) falam sobre mecanismos que podem explicar a relação do vírus com as comorbidades. O primeiro mecanismo que eles citam é a inflamação, seguido do estado hipercoagulativo e a ativação do sistema renina-angiotensina-aldosterona, como também a desregulação do sistema nervoso simpático. Uma vez que, o vírus faz uso da ECA2 para poder se ligar e invadir as células (HOFFMANN M, et al., 2020).

$\mathrm{Na}$ diabetes o sistema renina-angiotensina-aldosterona já está modificado, com maior produção de Angiotensina II (ANG II) que resulta em aumento na produção da espécie reativa de oxigênio (geradora de estresse oxidativo) e inflamação e causa diminuição da secreção e sensibilidade à insulina nos órgãos metabólicos (PATEL VB, et al., 2013). A elevação da ANG II acusada pela hiperglicemia, gera respostas patogênicas e estimulam a produção de moléculas de adesão e ativa os mecanismos de inflamação tecidual, e torna o organismo mais propenso a infecção, o que responde o maior comprometimento em casos de COVID-19, em que há alta taxa de inflamação e coagulação (HUSSAIN A, et al., 2020).

As células infectadas pelo COVID-19 e suas replicações causam as respostas inflamatórias, e ativação da morte celular programada, tudo por um meio de fácil infecção (TAY MZ, et al., 2020). Em 28 de junho, no mundo, 10.001.527 milhões de pessoas foram infectadas pelo COVID-19 com 499.124 mil óbitos (BOLETIM COVID-19, 2020). No Brasil, são 1,345.254 milhões de pessoas infectadas, com o primeiro caso registrado em 28 de fevereiro e 57.658 mil óbitos (CRODA J, et al., 2020). Em Sergipe já são 24.421 pessoas infectadas com 620 óbitos, tendo o primeiro registro de casos positivos e mortes em 14 de março de 2020, com taxa de letalidade em $2,5 \%$.

Houve uma maior incidência de óbitos na faixa etária de 80 anos ou mais para o sexo feminino, com 50 casos registrados (23,58\% do total), seguido de 60 a 69 anos para o sexo masculino, com 56 casos registrados (23,24\% do total) (BOLETIM COVID-19, 2020). No entanto, a prevalência das comorbidades não foi apresentada, assim como os óbitos com e sem comorbidades, além das correlações envolvendo a idade e sexo. Para melhor entendimento da influência das comorbidades para os óbitos em Sergipe se fez necessário o presente estudo, cujo objetivo foi evidenciar a prevalência e sua correlação com as comorbidades por idade e sexo dos óbitos confirmados por COVID-19 no estado de Sergipe-Brasil.

\section{MÉTODOS}

O estudo tratou-se de pesquisa retrospectiva e transversal de banco de dados brutos públicos em site, não havendo necessidade de aprovação do Comitê de Ética em Pesquisa com seres humanos. Assim, as buscas dos dados brutos do Estado de Sergipe foram consultadas na planilha de monitoramento diário de casos do Centro de Informações Estratégicas em Vigilância em Saúde (CIEVS) da Diretoria de Vigilância em Saúde (DVS); e do Gerenciador de Ambiente Laboratorial (GAL) da Diretoria de Vigilância Epidemiológica (DIVEP) subordinadas à Secretaria do Estado de Saúde (SES) do Estado de Sergipe, no dia 05/06/2020, às 13h, disponíveis no site: https://todoscontraocorona.net.br/boletim-covid-19-28-06-2020/. A busca contempla desde o primeiro caso de morte em 14 de março até o número acumulado das mortes confirmadas pelo COVID-19 em 28 de junho do ano de 2020 às 22 horas.

A análise estatística foi feita no software GraphPad Prism, os dados de prevalência foram apresentados em frequência absoluta e relativa, com análise estatística utilizando o Teste Qui-quadrado pela comparação da distribuição observada com a esperada. Para a correlação entre comorbidade e idade em ambos os sexos e por sexo masculino ou feminino dos óbitos confirmados foi utilizado o Teste de Spearman, considerando correlação fraca $(r=0,1$ a 0,3$)$, moderada $(r=0,4$ a 0,6$)$ e forte $(r=0,7$ a 1,0) e $p<0.05$ como resultado significativo.

\section{RESULTADOS}

A Secretaria de Estado da Saúde de Sergipe em 28 de junho de 2020 notificou 620 casos de óbitos confirmados para COVID-19. Dos 620 casos de óbitos, $453(73,06 \%)$ foram vinculados as comorbidades, 167 $(26,26 \%)$ sem comorbidades e $2(0,50 \%)$ sem diagnóstico. Dos 453 óbitos com comorbidades, 241 (53,2\%) eram do sexo masculino e $212(46,8 \%)$ do sexo feminino. Foram 56 municípios que registraram óbitos e 18

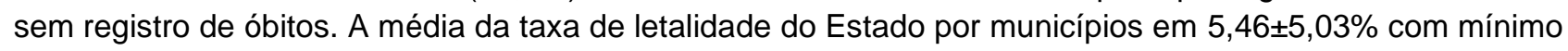
de $1,0 \%$ e máximo de $28,0 \%$ de letalidade. 
$\mathrm{Na}$ distribuição por frequência relativa desses óbitos por município, foi observado que $42,79 \%$ se encontram na capital (Aracaju); seguido do município de Nossa Senhora do Socorro com 10,2\%, São Cristovão com 6,2\%; e em seguida os municípios com menor frequência onde um (01) município apresentouse com cerca de 3,10\%, 03 municípios se apresentam com menos de 2,88\%; 10 municípios com 1\%; e por fim apresentando um índice menor que $1 \%$ o total de 36 municípios. Houve até 5 comorbidades para cada indivíduo, vinculadas aos óbitos, totalizando 736 ocorrências. Dessas ocorrências, 227(30,76\%) notificações para HAS; $208(28,18 \%)$ foram notificações para DM; e $85(11,52 \%)$ notificações para cardiopatias (Tabela 1). E ao estratificar as comorbidades por sexo foram: 389 notificações para o sexo masculino e 349 notificações para o sexo feminino. A prevalência maior para o sexo masculino foi para Hipertensão Arterial Sistêmica (HAS) com 119 (30,59\%); seguido da Diabetes Mellitus (DM) com cerca de 109 (28,02\%) e Cardiopatias 42(10,8\%). Já no sexo feminino a prevalência maior foi HAS com 108 (30,95\%), seguido por DM no com total de $99(28,37)$ e as Cardiopatias com 43 (12,32\%) (Tabela 1).

Tabela 1 - Prevalência das comorbidades por sexo dos óbitos positivos para COVID-19 no Estado de Sergipe até 28 de junho de 2020 .

\begin{tabular}{|c|c|c|c|c|}
\hline Comorbidades & $\begin{array}{l}\text { Todos } \\
\mathrm{N}(\%)\end{array}$ & $\begin{array}{c}\text { Masculino } \\
\mathrm{N}(\%)\end{array}$ & $\begin{array}{c}\text { Feminino } \\
\mathrm{N}(\%)\end{array}$ & Valor de $p$ \\
\hline Alzheimer & $10(1,35)$ & $6(1,54)$ & $4(1,14)$ & \\
\hline Amaurose & $1(0,13)$ & $1(0,25)$ & $0(0,00)$ & \\
\hline Anemia & $1(0,13)$ & $0(0,00)$ & $1(0,28)$ & \\
\hline Asma & $7(0,94)$ & $3(0,77)$ & $4(1,14)$ & \\
\hline Acidente Vascular Cerebral & $7(0,94)$ & $4(1,02)$ & $3(0,85)$ & \\
\hline Cardiopatias & $85(11,52)$ & $42(10,8)$ & $43(12,32)$ & \\
\hline Crise Convulsiva & $1(0,13)$ & $0(0,00)$ & $1(0,28)$ & \\
\hline Demência Senil & $2(0,27)$ & $1(0,25)$ & $1(0,28)$ & \\
\hline Diabetes Mellitus & $208(28,18)$ & $109(28,02)$ & $99(28,37)$ & \\
\hline Doença Hematológica & $2(0,27)$ & $1(0,25)$ & $1(0,28)$ & \\
\hline Doença Metabólica & $5(0,67)$ & $2(0,51)$ & $3(0,85)$ & \\
\hline Doença Neurológica & $5(0,67)$ & $3(0,77)$ & $2(0,57)$ & \\
\hline Doença Renal & $27(3,65)$ & $20(5,14)$ & $7(2,00)$ & \\
\hline $\begin{array}{c}\text { Doença Pulmonar Obstrutiva } \\
\text { Crônica }\end{array}$ & $24(3,25)$ & $12(3,08)$ & $12(3,43)$ & 0,001 \\
\hline Epilepsia & $1(0,13)$ & $0(0,00)$ & $1(0,28)$ & \\
\hline Grande Queimada & $1(0,13)$ & $0(0,00)$ & $1(0,28)$ & \\
\hline Hepatopatia & $9(1,22)$ & $7(1,79)$ & $2(0,57)$ & \\
\hline $\begin{array}{l}\text { Hipertensão Arterial } \\
\text { Sistêmica }\end{array}$ & $227(30,76)$ & $119(30,59)$ & $108(30,95)$ & \\
\hline Hipoacusia & $1(0,13)$ & $1(0,25)$ & $0(0,00)$ & \\
\hline Imunossupressão & $16(2,16)$ & $9(2,31)$ & $7(2,00)$ & \\
\hline $\begin{array}{c}\text { Insuficiência Respiratória } \\
\text { Aquda }\end{array}$ & $1(0,13)$ & $1(0,25)$ & $0(0,00)$ & \\
\hline Neoplasia & $30(4,06)$ & $15(3,85)$ & $15(4,29)$ & \\
\hline Obesidade & $45(6,09)$ & $18(4,62)$ & $27(7,73)$ & \\
\hline Parkinson & $2(0,27)$ & $2(0,51)$ & $0(0,00)$ & \\
\hline Pneumopatia & $6(0,81)$ & $4(1,02)$ & $2(0,57)$ & \\
\hline Politrauma & $1(0,13)$ & $1(0,25)$ & $0(0,00)$ & \\
\hline Prematuridade & $1(0,13)$ & $1(0,25)$ & $0(0,00)$ & \\
\hline Síndrome de Down & $2(0,27)$ & $1(0,25)$ & $1(0,28)$ & \\
\hline Síndrome de Menks & $1(0,13)$ & $1(0,25)$ & $0(0,00)$ & \\
\hline Tabagismo & $3(0,40)$ & $2(0,51)$ & $1(0,28)$ & \\
\hline Transtorno Psíquico & $2(0,27)$ & $0(0,00)$ & $2(0,57)$ & \\
\hline Tuberculose & $1(0,13)$ & $1(0,25)$ & $0(0,00)$ & \\
\hline Tumor Cerebral & $1(0,13)$ & $0(0,00)$ & $1(0,28)$ & \\
\hline Sem diagnóstico & $2(0,27)$ & $2(0,51)$ & $0(0,00)$ & \\
\hline Total & $738(100,00)$ & $389(100,00)$ & $349(100,00)$ & \\
\hline
\end{tabular}

Legenda: Considerando valor de $p<0,05 ; p<0,01$ e $p<0,001$. Teste Qui-quadrado.

Fonte: Almeida KC, et al., 2020. Baseado em SAÚDE, 2020. In: Boletim COVID-19. 
Em relação a prevalência de óbitos por faixa etária, tanto no sexo feminino como no masculino, a partir de 60 anos em diante verificou-se aumento na frequência relativa para óbitos pelo COVID-19. Foi encontrado no sexo masculino maior prevalência de $23,24 \%, 20,75 \%$ e 22,82\% nas faixas etárias de 60 a 69 anos, 70 a 79 anos e acima de 80 anos, Dados semelhantes foram encontrados no sexo feminino de $23,11 \%, 23,11 \%$ e 23,58\% nas respectivas faixas etárias (Tabela 2).

Tabela 2 - Prevalência de faixa etária por sexo dos óbitos positivos para COVID-19 no Estado de Sergipe até 28 de junho de 2020.

\begin{tabular}{lccccc}
\hline \multirow{2}{*}{ Idade } & \multicolumn{3}{c}{ Masculino } & \multicolumn{3}{c}{ Feminino } & Valor de $\boldsymbol{p}$ \\
\cline { 2 - 6 } & $\mathbf{N}$ & $\%$ & $\mathbf{N}$ & $\%$ & 0 \\
1 < ano & 2 & 0,82 & 0 & 0,47 \\
5 anos & 2 & 0,82 & 1 & 0,94 \\
14 anos & 2 & 0,82 & 2 & 0,47 & \\
$20-19$ anos & 1 & 0,41 & 1 & 7,07 & 0,001 \\
$40-49$ anos & 13 & 5,39 & 15 & 7,54 & \\
$50-59$ anos & 24 & 9,95 & 16 & 13,68 \\
$60-69$ anos & 36 & 14,94 & 29 & 23,11 \\
$70-79$ anos & 56 & 23,24 & 49 & 23,11 \\
$80+$ & 50 & 20,75 & 49 & 23,58 \\
\hline Total & 55 & 22,82 & 50 & 100
\end{tabular}

Legenda: Considerando valor de $p<0,05 ; p<0,01$ e $p<0,001$. Teste Qui-quadrado.

Fonte: Almeida KC, et al., 2020. Baseado em SAÚDE, 2020. In: Boletim COVID-19.

Ao analisar a correlação dessas comorbidades com a idade independente do sexo foi encontrada correlação moderada $(r=0,65 ; p=0,001)$ para os óbitos nas pessoas acima de 50 anos de idade, e correlação elevada $(r=0,8 ; p=0,001)$ para óbitos nas pessoas com mais de 60 anos. Na comorbidade HAS, tanto o sexo masculino como o feminino apresentaram baixa correlação $(r=0,18 ; p=0,003 ; r=0,23 ; p=0,001$ respectivamente). E quando houve associação das comorbidades HAS e DM essas permaneceram com correlação baixa, no masculino $(r=0,13 ; p=0,043)$ e feminino $(r=0,16 ; p=0,018)$ (Tabela 3).

Tabela 3 - Correlação entre idade e comorbidades Diabetes Mellitus, Hipertensão arterial Sistêmica e Cardiopatias por sexo dos óbitos positivos para COVID-19 no Estado de Sergipe até 28 de junho de 2020.

\begin{tabular}{|c|c|c|}
\hline Masculino/ Feminino & vs. Acima de 50 anos & vs. Acima de 60 anos \\
\hline Valor de $r$ & 0,6519 & 0,8047 \\
\hline Valor de $p$ & 0,0001 & 0,001 \\
\hline Idade & vs. Hipertensão Arterial Sistêmica & vs. Diabetes Mellitus \\
\hline \multicolumn{3}{|l|}{ Masculino } \\
\hline Valor de $r$ & 0,1858 & 0,05817 \\
\hline Valor de $p$ & 0,0039 & 0,3696 \\
\hline Masculino & \multicolumn{2}{|c|}{ vs. Hipertensão Arterial Sistêmica e Diabetes Mellitus } \\
\hline Valor de $r$ & \multicolumn{2}{|c|}{0,1303} \\
\hline Valor de $p$ & \multicolumn{2}{|c|}{0,0437} \\
\hline \multicolumn{3}{|l|}{ Feminino } \\
\hline Valor de $r$ & 0,2379 & 0,07966 \\
\hline Valor de $p$ & 0,0005 & 0,2481 \\
\hline Feminino & \multicolumn{2}{|c|}{ vs. Hipertensão Arterial Sistêmica e Diabetes Mellitus } \\
\hline Valor de $r$ & \multicolumn{2}{|c|}{0,1622} \\
\hline Valor de $p$ & \multicolumn{2}{|c|}{0,0181} \\
\hline
\end{tabular}

Legenda: Considerando valor de $p<0,05 ; p<0,01$ e $p<0,001$. Teste Spearman $r$, considerando correlação fraca $(r=0,1$ a 0,3$)$; moderada $(r=0,4$ a 0,6$)$ e forte $(r=0,7$ a 1,0$)$ com $p<0,05$.

Fonte: Almeida KC, et al., 2020. Baseado em SAÚDE, 2020. In: Boletim COVID-19. 


\section{DISCUSSÃO}

A pandemia causada pelo COVID-19 já matou mais de trezentos mil pessoas no mundo, 26 mil no Brasil e em Sergipe já são 143 pessoas até 28 de junho de 2020. Ainda em Sergipe foi percebido que os óbitos associados a faixa etária mais alta e as comorbidades prevaleceram, chegando até a 4 comorbidades em um único indivíduo.

Esses dados sugerem que ter uma ou mais comorbidades é fator de risco para óbito (HARAPAN H, et al., 2020). Ruan $Q$, et al. (2020), relatam que o número de comorbidades são fatores de risco para óbito por COVID-19 e Shahid Z, et al. (2020) reportam maior prevalência de morte nos indivíduos com mais de 65 anos. O que indica maior prevalência envolvendo indivíduos idosos e com comorbidade associada.

As comorbidades que mais se destacaram como preditor de óbitos da COVID-19 em Sergipe-Brasil, foram: HAS, DM seguido de cardiopatias. A prevalência para HAS ficou bem próximo ao estudo de Guan WJ, et al. (2019), com 23,70\% e bem menor que o estudo de Zhang JJ, et al. (2020) com 30\%.

A alta correlação da presença de HAS e DM nos indivíduos acometidos pelo COVID-19, tem gerado preocupação com medida terapêutica (medicamentos). A utilização de Inibidores da Enzima de Conversora de Angiotensina (IECAs) e Bloqueadores de Receptores de Angiotensina II (BRAs) tem mostrado supraregulação da ECA2. No entanto altos níveis da enzima, podem aumentar o risco de infecção pelo COVID-19, possivelmente explicado pelo polimorfismo apresentado pela ECA2 que pode ter ligação com a HAS e DM (FANG L, et al., 2020).

Em estudos retrospectivos, foi realizada a associação do uso de IECAs e BRAs na taxa de mortalidade, por quaisquer causas, em pacientes hipertensos hospitalizados pela COVID-19. Foram comparados indivíduos que utilizavam IECAs e BRAs com aqueles que não faziam uso dos medicamentos.

Yang G, et al., (2020), demonstraram baixo risco de mortalidade naqueles indivíduos que utilizavam as medicações. Zhang $P$, et al., (2020), também perceberam diminuição na taxa de mortalidade nos indivíduos que utilizavam IECAs e BRAs, quando comparado aos que faziam uso de outros medicamentos antihipertensivos.

Em revisão, Cheng $\mathrm{H}$, et al. (2020) especularam o comportamento do Sistema Renina Angiotensina (SRA), estritamente ligado aos níveis de pressão arterial. A ANG II sintetizada após ativação do SRA, promove respostas inflamatórias que podem ocasionar no aparecimento de edema pulmonar, comumente ligado a Síndrome Aguda do Desconforto Respiratório (SADR).

Em contraste, a Enzima Conversora de Angiotensina II (ECA2) é altamente expressa no tecido pulmonar durante processo de proteção de lesão aguda neste sistema. Entretanto, o vírus (2019-nCoV, SRAS-CoV-2) causador da COVID-19 parece ter como principal via de invasão do organismo os receptores primários da ECA2. Pode-se afirmar que, a diminuição dos níveis de ECA2 e aumento da ANG II pode causar lesão do sistema pulmonar, com aumento na permeabilidade vascular e presença de SADR (CHEN H, et al., 2020).

Os sítios que expressam a ECA2 são os mesmos órgãos que o COVID-19 infecta (células epiteliais do pulmão, intestino, rim e vasos sanguíneos). E é possível que o tratamento da HAS e DM com medicamentos que atuem aumentando a ECA2 ou bloqueadores dos receptores da ECA2 possam ajudar na replicação do COVID-19 com agravamento da infecção (WAN Y, et al., 2020; FANG L, et al., 2020).

Outros autores já propõem que esses indivíduos apresentam expressão baixa da ECA2 e a infecção por COVID-19 desregule ainda mais causando piores comprometimentos pulmonares e cardiovasculares (VERSMISSEN J, et al., 2020).

Sommerstein R, et al. (2020) elucidaram o possível efeito deletério causado pelos altos níveis expressos de ECA2 nos indivíduos com DM, que fazem uso de IECAs e BRAs. Apesar da alta expressão de ECA2 levar a possível proliferação infecciosa, o estudo citou que o uso de Inibidores do Sistema Renina-Angiotensina 
pode ser benéfico na SADR. Mas trata-se de urgência a necessidade de determinação da influência da supraregulação de ECA2 no curso da COVID-19.

Em relação a prevalência da DM de Sergipe-Brasil, os resultados foram mais altos quando comparados aos resultados de Yang X, et al. (2020) com 22\%. Prevalência bem maior que encontrada nos estudos de Guan WJ, et al. (2019), com 16,2\% e um pouco menor que os resultados de Deng SQ e Peng HJ (2019), que apresentaram a alta prevalência de $42,3 \%$. A diabetes como fator de risco para óbito por COVID-19 foi relatada por Gupta R, et al. (2020) e de forma semelhante foi encontrado no presente estudo.

Na revisão de literatura de Lippi G e Plebani M (2020), alterações laboratoriais foram encontradas em 08 estudos; no entanto, entre os vários achados alterados, o índice glicêmico elevado foi mencionado apenas no trabalho de Chen N, et al. (2019) e nenhum relato vinculado a HAS.

Entretanto, estudo em população com 72.314 casos confirmados para COVID-19 evidenciou aumento de mortalidade maior em pessoas com diabetes $(7,3 \%)$ em relação as comorbidades no geral $(2,3 \%)$, o que corrobora para os nossos resultados, quanto a população do estado de Sergipe-Brasil (WU Z e MCGOOGAN JM., 2019).

E em relação aos achados de cardiopatias de Sergipe-Brasil, a prevalência ficou abaixo do apresentado por Yang X, et al. (2020) com 22\% dos óbitos por COVID-19. A correlação das comorbidades para os óbitos do COVID-19 de acordo com o sexo e idade em Sergipe-Brasil, evidenciou que, tanto as mulheres quanto os homens apresentaram as comorbidades como preditores altos e moderados para óbito, e em específico, mais baixa em relação a HAS ou DM.

Ao associar as duas comorbidades essa correlação manteve-se baixa. Muniyappa R e Gubbi S (2020), chamam atenção para a população com perfil das comorbidades de HAS e DM, já que elas se destacaram também entre os óbitos por COVID-19 nos Estados Unidos da América.

No entanto, Tadic M, et al. (2020) em revisão observaram que há um alto nível de heterogeneidade nos estudos que correlacionam a DM com os óbitos por COVID-19, embora destaquem que a maior frequência de agravamento foi observada naqueles indivíduos com DM em comparação aos que não diagnosticados com a doença. Dados estes que complementam aos nossos achados, uma vez que, a população com DM esteve como a segunda maior prevalência nos casos hospitalizados por COVID-19 no estado de SergipeBrasil.

Na figura 1 a representação gráfica permite visualização da instalação do SARS-CoV-2 e algumas de suas repercussões, após ligar-se ao receptor da ECA2 e promover cascata, com diminuição dos níveis de ECA2 e aumento dos níveis de ANG II, leva a indução de processos inflamatórios (Chen N, et al., 2019) que contribuem para agravamento do quadro de infecção viral em indivíduos com HAS/DM. No entanto, como discutido, a utilização dos IECAs ou BRAs pode levar o processo por um percurso menos deletério ao aumentar os níveis de ECA2 e produzir um efeito reparador ao reduzir o processo inflamatório nos pulmões (JIA H, 2016).

O mecanismo de ação (Figura 1) dos IECAs se baseia na inibição da Enzima Conversora de Angiotensina (ECA), responsável por transformar a Angiotensina I (ANG I) em ANG II. Ao ligar-se ao Receptor de Angiotensina (RA) a ANG II promove cascata pró-inflamatória, que pode comprometer o sistema pulmonar, como discutido anteriormente.

Os BRAs (Figura 1) tem efeito sobre os RAs, após inibir esses receptores, tem seu efeito indireto sobre a ANG II, impossibilita sua ligação aos RAs e limita os efeitos de possíveis processos inflamatórios. A utilização destes medicamentos parece gerar efeito reverso ao apresentado pela instalação do SARS-CoV-2, ao promover elevação dos níveis de ECA2, além de atuar na redução da pressão arterial, reduzir as respostas inflamatórias e proteger o sistema cardiovascular de possíveis lesões (Figura 1) (VADUGANATHAN M, et al., 2020). 
Figura 1 - Representação gráfica da ligação do vírus SARS-CoV-2 no receptor da ECA2 e os efeitos após interação dos medicamentos (IECA, BRAs) utilizados pelos indivíduos com HAS/DM.

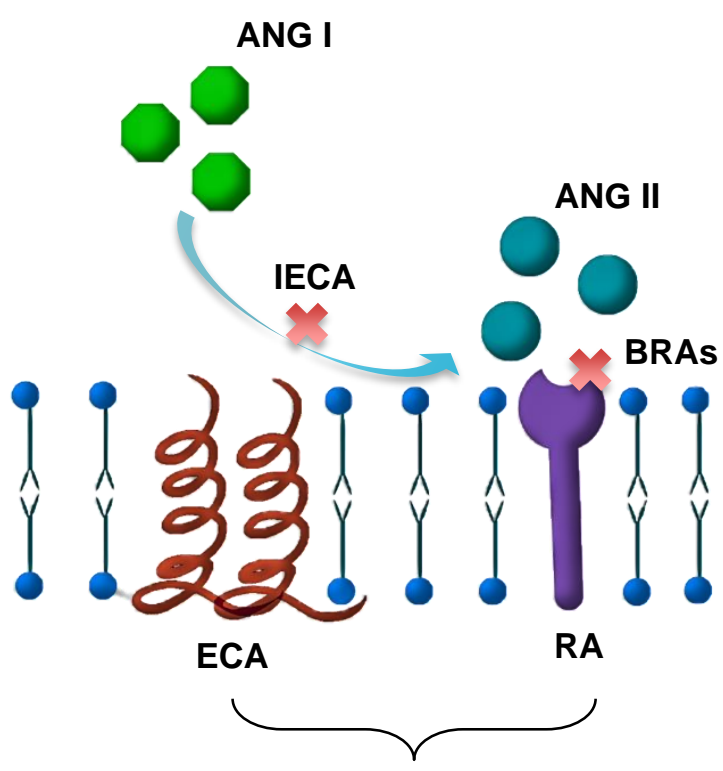

Inibição da ECA / Bloqueio do RA

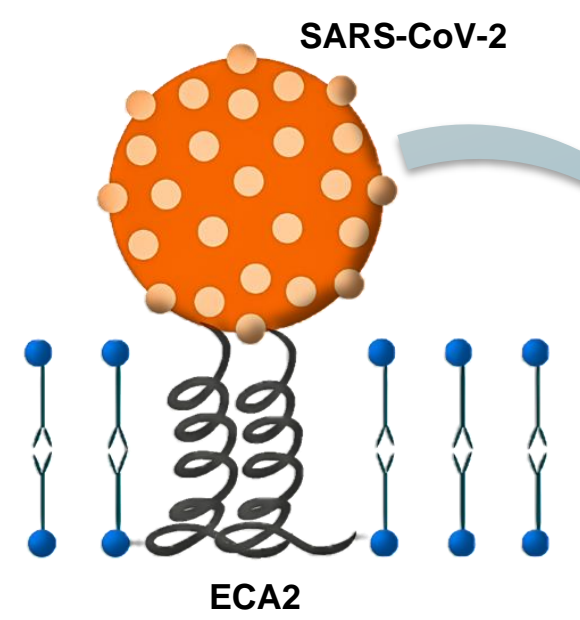

Níveis de ECA2

Níveis de ANG II

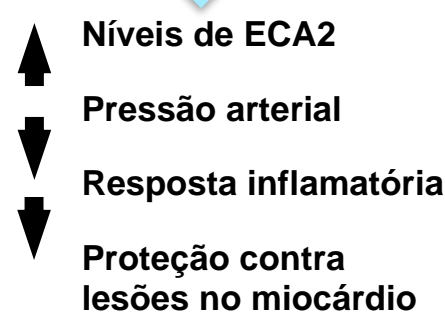

\section{Permeabilidade capilar pulmonar \\ Edema pulmonar \\ SADR}

\section{Hipertensão arterial}

Legenda: ANG I: Angiotensina I; IECA: Inibidores da Enzima Conversora de Angiotensina; ANG II: Angiotensina II; BRAs: Bloqueadores de Receptores de Angiotensina; ECA: Enzima Conversora de Angiotensina; RA: Receptor de Angiotensina; ECA2: Enzima Conversora de Angiotensina II; SADR: Síndrome Aguda do Desconforto Respiratório. Fonte: Almeida KC, et al., 2020.

Como apresentado nos resultados do presente estudo, a maior prevalência de indivíduos hospitalizados por COVID-19 em Sergipe-Brasil está relacionada a presença pregressa de HAS e DM. Diante disto, os indivíduos necessitam de assistência terapêutica medicamentosa. Como discutido anteriormente, há uma posição controvérsia entre benefícios ou não das drogas utilizadas para tratamento desses indivíduos, embora a continuidade no tratamento seja necessária para regulação da homeostase e prevenção de possíveis episódios de descompensação.

De acordo com isso, SINGH AK, et al., (2020) abordaram em revisão, a utilização dos variados medicamentos utilizados por indivíduos diagnosticados com COVID-19, com comorbidades associadas. Acerca daqueles que apresentam HAS e DM, a recomendação prática baseada nas evidências é de que haja continuação das medidas terapêuticas, como também propomos. Tanto o tratamento da DM como da HAS foram citados com possíveis envolvimentos ou não com a gravidade do COVID-19, assim como a própria HAS e DM (GUPTA R, et al., 2020). Nesse entendimento se faz necessário levantamentos de parâmetros clínicos e bioquímicos para entender como a hiperglicemia, a hiperinsulinemia e os agentes hipoglicêmicos afetam a patogênese do COVID-19, principalmente de mulheres com mais idade, como mostra os resultados deste presente estudo. 


\section{CONCLUSÃO}

Conclui-se que os resultados em Sergipe-Brasil se assemelham com a problemática mundial em relação às comorbidades preditoras dos óbitos por COVID-19. Os óbitos dos indivíduos diagnosticados e hospitalizados por COVID-19 no estado de Sergipe-Brasil apresentam correlação com a presença de comorbidades, sendo a HAS a comorbidade prevalente, seguida da DM e cardiopatias, em indivíduos de ambos os sexos, e naqueles acima dos 60 anos. O estudo contribui para o entendimento de que, a evolução dos casos e as ideias ainda não robustas acerca da, influência do tratamento medicamentoso de HAS e DM nos indivíduos com COVID-19, podem tornar-se um problema de saúde pública.

\section{REFERÊNCIAS}

1. ALMEIDA AT, et al. Impacts of a Brazilian pharmaceutical program on the health of cronic patients. Revista de Saúde Pública, 2019; 01-11.

2. BULUT C, KATO Y. Epidemiology of COVID-19. Turkish journal of medical sciences, 2020; 50: 563-570.

3. CHEN N, et al. Epidemiological and clinical characteristics of 99 cases of 2019 novel coronavirus pneumonia in Wuhan, China: a descriptive study. The Lancet, 2020; 395: 507-513.

4. CHENG H, et al. Organ-protective effect of Angiotensin-Converting Enzyme 2 and its effect on the prognosis of COVID-19. Journal of Medical Virology, 2020; 92: 726-730.

5. CRODA J, et al. COVID-19 in Brazil: advantages of a socialized unified health system and preparation to contain cases. Revista da Sociedade Brasileira de Medicina Tropical, 2020; 53: 1-6.

6. DENG SQ, PENG HJ. Characteristics of and public health responses to the Coronavirus disease 2019 outbreak in China. Journal of Clinical Medicine, 2020; 9(2): 1-10.

7. FANG L, et al. Are patients with hypertension and diabetes mellitus at increased risk for COVID-19 infection. Lancet Respiratory Medicine, 2020; 8: 21.

8. $\mathrm{GE} \mathrm{H}$, et al. The epidemiology and clinical information about COVID-19. European Journal of Clinical Microbiology \& Infectious Diseases, 2020; 39:1011-1019.

9. GUAN WJ, et al. Clinical characteristics of Coronavirus disease 2019 in China. The New England Journal of Medicine, 2020; 328: 1708-1720.

10. GUPTA R, et al. Clinical considerations for patients with diabetes in times of COVID-19 epidemic. Diabetes \& Metabolic Syndrome: Clinical Research \& Reviews, 2020; 14: 211-212.

11. HARAPAN H, et al. Coronavirus disease 2019 (COVID-19): A literature review. Journal of Infection and Public Health, 2020; 13: 667-673.

12. HENRY BM, et al. Hematologic, biomechanical and immune biomarker abnormalities associated with severe illness and mortality in coronavirus disease 2019 (COVID-19): a meta-analysis. Clinical Chemistry and Laboratory Medicine, 2020; 58: 1021-1028.

13. HOFFMANN M, et al. SARS-CoV-2 Cell Entry Depends on ACE2 and TMPRSS2 and Is Blocked by a Clinically Proven Protease Inhibitor. Cell, 2020; 181: 271-280.

14. HUSSAIN A, et al. COVID-19 and diabetes: Knowledge in progress. Diabetes Research and Clinical Practice, 2020; 162: 01-09.

15. JIA H. Pulmonary Angiotensin-Converting Enzyme 2 (ACE2) and Inflamatory Lung Disease. Shock, 2016; 46(3): 239248.

16. JIN Y, et al. Virology, epidemiology, pathogenesis, and control of COVID-19. Viruses, 2020;12(4): 372

17. LI LQ, et al. COVID-19 patients' clinical characteristics, discharge rate, and fatality rate of meta-analysis. Journal of Medical Virology, 2020; 92: 577-583.

18. LI W, et al. Angiotensin-converting enzyme 2 is a functional receptor for the SARS coronavirus. Nature, 2003; 426 : 450-454.

19. LIPPI G, PLEBANI M. Laboratory abnormalities in patients with COVID-2019 infection. Clinical Chemistry and Laboratory Medicine, 2020; 58(7): 1131-1134.

20. LIU Z, et al. Composition and divergence of coronavirus spike proteins and host ACE2 receptors predict potential intermediate hosts of SARS-CoV-2. Journal of Medical Virology, 2020: 01-07

21. MENG J, et al. Renin-Angiotensin System Inhibitors improve the clinical outcomes of COVID-19 patients with hypertension. Emerging Microbes \& Infections, 2020; 9: 757-760.

22. MINISTÉRIO DA SAÚDE. Plano de ações estratégicas para o enfrentamento das Doenças Crônicas não transmissíveis (DCNT) no Brasil 2011-2022. 2011; 01-160.

23. MUNIYAPPA R, GUBBI S. COVID-19 pandemic, coronaviruses, and diabetes mellitus. American Journal of Physiology Endocrinology and Metabolism, 2020; 318:736-741.

24. PATEL VB, et al. Role of angiotensin-converting enzyme 2 (ACE2) in diabetic cardiovascular complications. Clinical Science, 2013; 126: 471-482.

25. RODRIGUES-MORALES AR, et al. Clinical, laboratory and imaging features of COVID-19: A systematic review and meta-analysis. Travel Medicine and Infectious Disease, 2020; 34: 1-13. 
26. RUAN Q, et al. Clinical predictors of mortality due to COVID-19 based on an analysis of data of 150 patients from Wuhan, China. Intensive Care Medicine, 2020; 46: 846-848.

27. SAÚDE. 2020. In: Boletim COVID-19. Centro de Operações de Emergências em Saúde Pública. Informe Epidemiológico de 05 de junho de 2020. Centro de Informações Estratégicas em Vigilância em Saúde (CIEVS) da Diretoria de Vigilância em Saúde (DVS) subordinada a Secretaria de Estado da Saúde (SES). Disponível em: www.todoscontraocorona.net.br. Acesso em: 5 Jun. 2020.

28. SINGH AK, et al. Diabetes in COVID-19: Prevalence, pathophysiology, prognosis and practical considerations. Diabetes \& Metabolic Syndrome: Clinical Research \& Reviews, 2020; 14: 303-310.

29. SOMMERSTEIN R, et al. Coronavírus Disease 2019 (COVID-19): Do Angiotensin-Coverting Enzyme Inhibitors/Angiotensin Receptor Blockers have a biphasic effect? Journal of the American Heart Association, 2020; 9: 1-4.

30. TADIC M, et al. COVID-19 and diabetes: Is there enough evidence? The Journal of Clinical Hypertension, 2020; 22: 943-948.

31. TAY MZ, et al. The Trinity of COVID-19: imunnity, inflamation and intervention. Nature Reviews, 2020; 363-374.

32. VADUGANATHAN M, et al. Renin-Angiotensin-Aldosterone System Inhibitors in Patients with Covid-19. The New England Journal of medicine, 2020; 382: 1653-1659.

33. VERSMISSEN J, et al. Angiotensin-converting enzyme-2 in SARS-CoV-2 infection: good or bad. Journal of Hypertension, 2020; 38: 1196-1197.

34. WAN Y, et al. Receptor recognition by the novel coronavirus from Wuhan: analysis based on decade-long structural studies of SARS coronavirus. Journal of Virology, 2020; 94: 1-9.

35. WU Z, MCGOOGAN JM. Characteristics of and important lessons from the Coronavirus disease 2019 (COVID-19) outbreak in China: summary of a report of 72314 cases from the Chinese center for disease control and prevention. Jama Network, 2020; 323: 1239-1242.

36. YANG G, et al. Effects of Angiotensin II Receptor Blockers and ACE (Angiotensin-Converting Enzyme) Inhibitors on Virus Infection, Inflammatory Status, and Clinical Outcomes in patients with COVID-19 and Hypertension: A SingleCenter Restrospective Study. Hypertension, 2020; 76: 51-58.

37. YANG X, et al. Clinical course and outcomes of critically ill patients with SARS-CoV-2 pneumonia in Wuhan, China: a single-centered, retrospective, observational study. Lancet Respiratory Medicine, 2020; 8: 475-482.

38. ZHANG JJ, et al. Clinical characteristics of 140 patients infected with SARS-CoV-2 in Wuhan, China. Allergy, 2020; 75: $1730-1741$.

39. ZHANG P, et al. Association of inpatient use of Angiotensin-Converting Enzyme Inhibitors and Angiotensin II Receptor Blockers with mortality amongpatients with Hypertensionhospitalized with COVID-19. Circulation Research, 2020; 126: 1671-1681.

40. ZHOU F, et al. Clinical course and risk factors for mortality of adult inpatients with COVID-19 in Wuhan, China: a retrospective cohort study. The Lancet, 2020; 395: 1054-1062. 